\title{
Wavefront reconstruction algorithms and simulation results for multiconjugate adaptive optics on giant telescopes
}

\section{Brent L. Ellerbroek}

Brent L. Ellerbroek, "Wavefront reconstruction algorithms and simulation results for multiconjugate adaptive optics on giant telescopes," Proc. SPIE 5382, Second Backaskog Workshop on Extremely Large Telescopes, (7 July 2004); doi: 10.1117/12.566342

SPIE Event: Second Backaskog Workshop on Extremely Large Telescopes, 2003, Backaskog, Sweden 


\title{
Wavefront reconstruction algorithms and simulation results for multi-conjugate adaptive optics on giant telescopes
}

\author{
Brent L. Ellerbroek \\ AURA New Initiatives Office, 950 N. Cherry Ave., Tucson AZ, 85719
}

\begin{abstract}
The very high-order multi-conjugate adaptive optics (MCAO) systems proposed for future giant telescopes will require new, computationally efficient, concepts for wavefront reconstruction. Advanced methods from computational linear algebra have recently been applied to this problem, and explicit simulations of MCAO wavefront reconstruction problems for 30-meter class telescopes are now possible using desktop personal computers. In this paper, we present sample simulation results obtained using these techniques to illustrate the trends in MCAO performance as the telescope aperture diameter increases from 8 to 32 meters. We consider systems based upon natural guidestars, sodium laser guidestars, and Rayleigh laser guidestars. The performance achieved by the first two classes of guidestars is similar, and the variation in their performance with respect to telescope size is very gradual over this range of aperture diameters. Next, we describe work in progress to adapt the minimum variance reconstruction algorithm, which is optimized for open-loop wavefront estimation, to the more realistic and meaningful case of closed-loop wavefront control. Finally, we summarize the current status of efforts to quantify the impact of sodium laser guide star (LGS) elongation on guidestar signal requirements for LGS AO systems on 30 meter class telescopes.
\end{abstract}

Keywords: Extremely large telescopes, multi-conjugate adaptive optics, wavefront reconstruction

\section{INTRODUCTION}

Multi-conjugate adaptive optics (MCAO) is a relatively new and still largely untested concept for widening the corrected field of view (FOV) of an astronomical adaptive optics (AO) system. ${ }^{1}$ In principle, wide-field atmospheric turbulence compensation is accomplished by means of multiple deformable mirrors (DMs) that apply phase adjustments in two or more planes optically conjugate to distinct ranges along the propagation path. The information to drive these DMs is obtained from wavefront sensor (WFS) measurements of multiple natural- or laser guidestars via a tomographic wavefront reconstruction algorithm. MCAO is now routinely invoked as an essential capability for future extremely large telescopes (ELTs), but the design approaches to be utilized and the levels of performance that may be achieved remain the subjects of extensive discussion. ${ }^{2-5}$ The range of system parameters debated includes the type and number of guidestars to employ; the associated wavefront sensing technology; the number, order, and conjugate ranges of the deformable mirrors; control bandwidth requirements; guidestar signal level requirements; and (assuming that they are used) how to manage the impact of sodium guidestar elongation. Defining, and defending, values for these parameters is challenging because of the very high-order MCAO systems that will be required for ELTs with aperture diameters of 20 meters or more.

The current MCAO system designs for 8-meter class telescopes are signficantly better developed, ${ }^{6-8}$ in part because of the availabilty of detailed simulation models to evaluate system performance as a function of the above first-order design parameters and a wide range of higher-order effects and implementation error sources. ${ }^{9}$ Using parallel computing architectures, the time required to simulate an MCAO system is not that much greater than for a classical AO system with only a single WFS and DM.10 Most of the features of these MCAO simulations scale reasonably well from an aperture diameter $D$ of 8 meters to 30 meters or even larger, with the noticable exception of the wavefront reconstruction algorithm that is used to determine DM actuator commands from the WFS measurements. Assuming that the DM actuator and WFS subaperture densities

Further author information: B.L.E.: E-mail: brent@noao.edu, Telephone: 1 (520) 318-8589, Fax: 1 (520) 318-8590 
are held constant, the computational burden to evaluate the reconstruction algorithm scales as $D^{6}$ using the standard computational techniques. This corresponds to a factor of 4096 as $D$ increases from 8 to 32 meters. Performing trade studies and exploring parameter space becomes impractical at this level of computation, assuming that wavefront reconstruction algorithms may be computed at all.

The last two years have fortunately seen significant progress in developing more computationally efficient approaches. Advanced techniques from computational linear algebra enable wavefront reconstructor to be evaluated without the explicit matrix multiplications and inversions that drive the cost of standard techiques. ${ }^{11-13}$ The computer time required for these new methods now scale as $D^{3}$, and the memory requirements are also small enough that MCAO wavefront reconstruction problems for system configurations with at least $8000 \mathrm{DM}$ actuators and 16000 WFS subapertures can be solved on desktop personal computers. ${ }^{14}$

In the last year we have begun using these methods to study MCAO performance issues on ELTs, at least for the limit case of an ideal system that is not degraded by time delay or WFS measurement noise. Some of these issues include (i) how performance varies as a function of aperture diameter with all other parameters held constant, (ii) Strehl ratio uniformity across the field-of-view, and (iii) sample point spread funtions. Sample results on these topics are summarized in sections 2 through 4.

Work is now commencing to study the next layer of MCAO wavefront reconstruction issues, such as evaluating and optimizing reconstructor performance for a closed-loop AO system. The classical mimimum variance (MV) wavefront reconstructor that provides optimum performance for MCAO is derived to minimize the residual wavefront error for an ideal open-loop AO system, in which the DM actuators respond immediately to an instantaneous snapshot of the uncorrected atmospheric turbulence. This approach is not necessarily optimal, or even stable, for the more realistic case of a closed-loop AO system in which the DM responds incrementally to WFS measurements that have been partially corrected by the previous set of actuator commands. All of the classical methods with which we are familiar for adapting the MV reconstructor to closed-loop operation have computation requirements that scale as $D^{6}$, and they are consequently impractical for the case of ELTs. At this point, we have developed at least one new approach that achieves stable results in closed-loop simulations. ${ }^{15}$ Sample results are presented in section 5.

Finally, section 6 summarizes work in progress on one possible approach to minimizing the impact of sodium laser guidestar elongation on WFS measurement accuracy for ELTs. The angular elongation of the image of a sodium laser guidestar in a Shack-Hartmann WFS is proportional to the linear separation $s$ between the laser transmitter and the wavefront sensing subaperture. This effect is already noticable for the Keck LGS AO system where $s=10 \mathrm{~m}$ for the edge subapertures, ${ }^{16}$ and even larger separations will be unavoidable for sodium LGS AO systems on ELTs. Shack-Hartmann wavefront sensing with quadrant detectors will no longer be practical, since the LGS signal required for equivalent tilt measurement accuracy would increase as (roughly) the second power of the spot elongation. Some of the approaches that have been suggested to defeat this effect include: (i) high resolution WFS focal planes and correlation-based wavefront sensing algorithms borrowed from solar AO systems, (ii) multiple laser beams with more than one launch telescope for each guide star, and (iii) wavefront sensors that dynamically refocus on a $1 \mathrm{~km}$ (or shorter) laser pulse as it propagates up through the sodium layer. We have begin studying approach (i) to quantify the improvement in WFS sensitivity, and the resulting reduction in LGS signal requirements, than may be possible with a high resolution WFS focal plane. The improvement is non-trivial, but LGS signal levels from 2.25 to 3 times greater than are presently planned for 8-meter class LGS AO systems would still be necessary using this approach. The noise-optimal pixel size for the "high resolution" WFS focal plane is approximately 1 arc second for CCD arrays with detector read noise of from 3 to 5 electrons.

This ongoing work on computationally efficient closed-loop wavefront reconstructors and improved WFS concepts for defeating LGS spot elongation are the last remaining technical challenges to be solved before detailed wave-optics propagation simulations of MCAO systems on 30-meter class ELTs will be feasible. Based upon the rate of recent progress, there is reason to hope that first results from these simulations may be available relatively quickly. 


\section{SAMPLE MCAO SYSTEM CONFIGURATION}

The new, computationally efficient algorithms that enable MCAO wavefront reconstruction problems for ELTs to be solved on desktop personal computers have already been described at several levels of detail in a number of previous papers. ${ }^{11-14}$ We consequently omit this material, and skip directly to a description of the sample observing scenario and MCAO system configuration used to generate the simulation results presented here.

Table 1 summarizes the atmospheric turbulence profile used for these simulations. This profile is a six-layer fit to thermosonde and generalized SCIDAR measurements collected at Cerro Pachon, Chile, the site of the Gemini-South telescope. ${ }^{17}$ The profile has been scaled to obtain a $r_{0}$ of $16 \mathrm{~cm}$ at a wavelength of $0.5 \mu \mathrm{m}$, which corresponds to roughly median seeing. The value of the isoplanatic angle $\theta_{0}$ is $2.65 \mathrm{arc} \mathrm{sec}$, or $12.85 \mu \mathrm{rad}$. The wind velocities have been selected to match the general shape of the median wind profile at Cerro Pachon. They provide a representative Greenwood Frequency, $f_{g}$, of $27.5 \mathrm{~Hz}$.

Finally, the turbulence outer scale $L_{0}$ is on the order of 32 meters, since periodic phase screens with widths of 64 meters are used in the simulations.

The representative AO parameters for the 8-to-32 meter MCAO systems tested against this atmospheric profile are, for the most part, related to the baseline design for a MCAO system at Gemini-South. ${ }^{6}$ The science FOV to be corrected is a one-arc-minute square. AO system performance is sampled at the center, edges, and corners of this square field, and the field-averaged residual mean-square phase error is estimated by combining the errors at these nine evaluation points using Simpson's rule in two dimensions. Three deformable mirrors are located conjugate to ranges of $0.0,5.15$, and $10.30 \mathrm{~km}$. The interactuator spacings on the three mirrors are equivalent to $0.5,0.5$, and $1.0 \mathrm{~m}$, respectively, at the telescope primary mirror.

The three DM's are controlled using measurements from five higher-order wavefront sensors viewing five guidestars at the center and four corners of a square FOV. For the simulations described in section 3 the size of this square FOV matches the 1 arc minute square science FOV. (For the simulations in sections 4 and 5 with $D=32 \mathrm{~m}$, the 4 outer guidestars have been displaced outwards to a distance of $\sqrt{2} \cdot 46=65$ arc sec from the center of the field to provide better sampling of the higher-altitude atmospheric turbulence and improve the uniformity of the wavefront correction.) The subaperture width for each WFS is equivalent to $0.5 \mathrm{~m}$ at the telescope primary mirror. We consider natural guidestars, sodium laser guidestars at a range of $90 \mathrm{~km}$, and Rayleigh laser guidestars at a range of $30 \mathrm{~km}$. Four natural guide stars located at the midpoints of the edges of the field are also included for full aperture tip/tilt sensing in the two LGS MCAO configurations, since the full aperture tilt modes of the LGS WFS measurements are unreliable due to the LGS position uncertainty problem.

The total number of higher-order WFS measurements varies from 2240 to 33320 as the aperture diameter increases from 8 to 32 meters. The total number of DM actuators ranges from 789 to 8449 .

The RMS WFS measurement noise is 0.02 arc sec, small enough to be almost negligible. The WFS sampling rate is $800 \mathrm{~Hz}$ for the dynamic closed-loop simulations presented in section 5 . The type I servo used to filter the DM actuator commands has a $-3 \mathrm{~dB}$ bandwidth of about $30 \mathrm{~Hz}{ }^{15}$

Table 1. Atmospheric turbulence profile used for AO simulations. See the text of section 2 for futher details.

\begin{tabular}{|r|r|r|r|}
\hline Layer & Altitude, $\mathrm{km}$ & Layer weight & Windspeed, $\mathrm{m} / \mathrm{s}$ \\
\hline 1 & 0.00 & 0.652 & 5.0 \\
2 & 2.58 & 0.172 & 13.0 \\
3 & 5.16 & 0.055 & 20.0 \\
4 & 7.73 & 0.025 & 30.0 \\
5 & 12.89 & 0.074 & 20.0 \\
6 & 15.46 & 0.022 & 10.0 \\
\hline
\end{tabular}




\section{TRENDS WITH APERTURE DIAMETER}

Fig. 1 illustrates the trends in MCAO system performance as a function of telescope aperture diameter with all of the remaining parameters listed in section 2 above held constant. "System performance" is quantified as the RMS residual OPD averaged over the telescope aperture diameter and the 1 arc minute square science FOV. The three curves in the figure correspond to MCAO systems employing natural guidestars, sodium laser guidestars, or Rayleigh laser guidestars at an altitude of $30 \mathrm{~km}$. The results obtained with the NGS MCAO system are virtually independent of aperture diameter, and the RMS residual OPD actually decreases very slightly from 140 to 137 nanometers as the telescope aperture diameter increases from 8 to 32 meters. The performance achieved by sodium LGS MCAO is somewhat poorer, with the RMS residual OPD varying from 149 to 159 nanometers over the same range of aperture diameters. We infer that the ensemble of 5 sodium laser guidestars sample the 3-dimensional turbulence volume well enough to compensate for most of the cone effect (or focus anisoplanatism) present in each individual LGS WFS measurement. In contrast, the performance of the Rayleigh LGS MCAO system degrades from 184 to 501 nanometers RMS as the aperture diameter $D$ increases from 8 to 32 meters, indicating that more guidestars would be necessary to accurately sample the full volume of atmospheric turbulence for the larger aperture system.

It is difficult to quantitatively extrapolate these results to even larger apertures, but we predict that (i) the performance of the NGS MCAO system would continue to improve very gradually, and that (ii) the performance of the sodium LGS MCAO would eventually begin to degrade more rapidly above some aperture diameter that we are unable to specify.

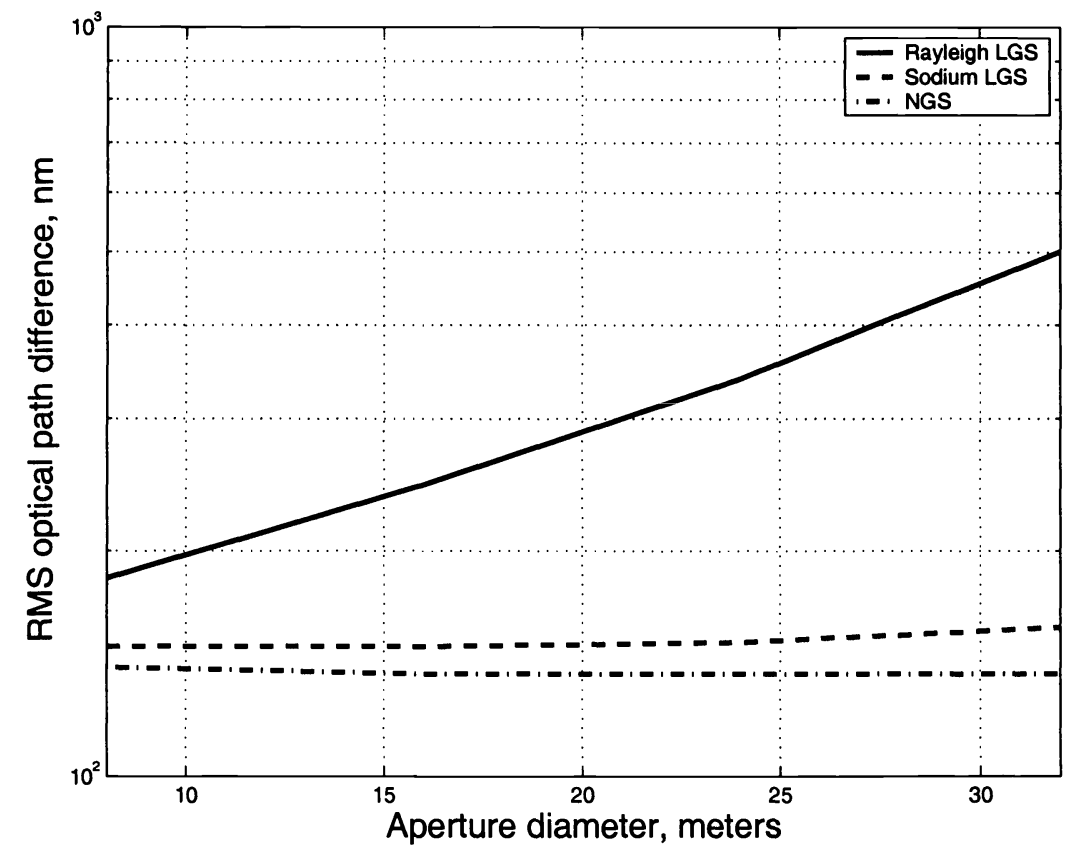

Figure 1. MCAO performance variations with aperture diameter

This figure plots the aperture- and field-averaged RMS residual OPD as a function of telescope aperture diameter for NGS, Sodium LGS, and Rayleigh LGS MCAO systems, with the remaining system parameters as described in section 2 above. 


\section{STREHL RATIOS AND POINT SPREAD FUNCTIONS}

The simulated residual OPDs generated by these simulations can be used to compute point spread functions (PSFs) and Strehl ratios by means of the usual Fresnel diffraction integral. Table 2 summarizes long exposure Strehl ratios in the J, H, and $\mathrm{K}$ spectral bands (i.e., wavelengths of $1.25,1.65$, and $2.20 \mu \mathrm{m}$ ) for sodium LGS MCAO systems with aperture diameters of 8 and 32 meters. Results are tabulated for an array of 3 by 3 evenly spaced points in one quadrant of the 1 arc minute square FOV-the array of points with coordinates $\theta_{x}=[0.00,0.25,0.50]$ arc sec and $\theta_{y}=[0.00,0.25,0.50]$ arc sec relative to the center of the field. The numerical values are computed as averages over 100 independent simulation trials. The Strehl ratios are relatively uniform, with some increase [decrease] at the very center [corner] of the field. There is no apparent loss of uniformity for the larger aperture diameter.

Whereas Strehl ratio uniformity will be important for applications of MCAO to multi-object spectroscopy, the finer details of the MCAO-corrected PSFs will be significant for imaging crowded fields. We have just begin to compute sample PSFs from simulated wavefronts, in part for the sake of curiousity. Fig. 2 plots grayscales and one-dimensional profiles through $\mathrm{H}$ band PSFs at the corner of the 1 arc minute square field for sodium LGS MCAO systems with aperture diameters of 8 and 32 meters. The images are 10.89 arc seconds across, and at this scale the principle PSF feature is the AO-corrected central core superimposed upon the residual seeing halo. Fig. 3 plots the central 0.68 arc second region of the PSFs to better illustrate the improvement in peak intensity and resolution achieved with the 32 meter aperture.

At this point, we are still waiting to include the impact of the remaining first-order adaptive optics error sources (noise and servo lag) in these PSFs before using them to generate simulated imagery. We believe that the very low-level structure observable in the $D=32 \mathrm{~m}$ PSF is a genuine consequence of the imperfections of the tomographic wavefront reconstruction algorithm, since these features vary across the field and are reduced by another five astronomical magnitudes in the on-axis PSF.

\section{CLOSED-LOOP TEMPORAL DYNAMICS}

All of the simulation results presented in sections 3 and 4 above are for the optimistic case of an idealized MCAO system that reconstructs and immediately corrects turbulence-induced phase errors based upon instantaneous snapshots of uncorrected, or open-loop, WFS measurements. These simplifying assumptions enable the use of minimum variance (MV) wavefront reconstruction algorithms, which are derived using a priori knowledge of atmospheric turbulence statistics and provide a useful upper bound upon the performance of any other wavefront reconstructor. However, practical real-world $\mathrm{AO}$ systems to date have operated in closed-loop, where incremental adjustments to the DM actuator commands are computed incrementally from WFS measurements of the residual phase errors still remaining after the preceding DM figure has been applied. The classical MV wavefront reconstructor is not necessarily optimal for this case, and in fact may not even be stable. The fundamental difficulty is that the MV wavefront reconstructor is optimized to estimate uncorrected turbulence

Table 2. Sodium LGS MCAO Strehl ratio uniformity for 8 and 32 meter aperture diameters. These results were obtained for the atmospheric turbulence profile and MCAO system parameters summarized in section 2 above. Results are tabulated for an array of 3 by 3 evenly spaced points in one quadrant of the system's 1 arc minute square FOV-the array of points with coordinates $\theta_{x}=[0.00,0.25,0.50]$ arc sec and $\theta_{y}=[0.00,0.25,0.50]$ arc sec relative to the center of the field.

\begin{tabular}{|c|c|c|c|c|c|c|c|c|c|}
\cline { 2 - 9 } \multicolumn{1}{c|}{} & \multicolumn{3}{|c|}{ J band } & \multicolumn{3}{c|}{$\mathrm{H}$ band } & \multicolumn{3}{c|}{ K band } \\
\hline \multirow{3}{*}{$D=8 \mathrm{~m}$} & 0.517 & 0.495 & 0.467 & 0.685 & 0.668 & 0.645 & 0.808 & 0.797 & 0.781 \\
\cline { 2 - 10 } & 0.541 & 0.498 & 0.495 & 0.703 & 0.669 & 0.668 & 0.820 & 0.798 & 0.797 \\
\cline { 2 - 9 } & 0.661 & 0.541 & 0.517 & 0.788 & 0.703 & 0.685 & 0.875 & 0.820 & 0.808 \\
\hline \multirow{3}{*}{$D=32 \mathrm{~m}$} & 0.487 & 0.462 & 0.401 & 0.661 & 0.641 & 0.591 & 0.792 & 0.778 & 0.744 \\
\cline { 2 - 9 } & 0.494 & 0.472 & 0.462 & 0.667 & 0.649 & 0.641 & 0.796 & 0.784 & 0.778 \\
\cline { 2 - 9 } & 0.561 & 0.494 & 0.487 & 0.717 & 0.667 & 0.661 & 0.829 & 0.796 & 0.792 \\
\hline
\end{tabular}



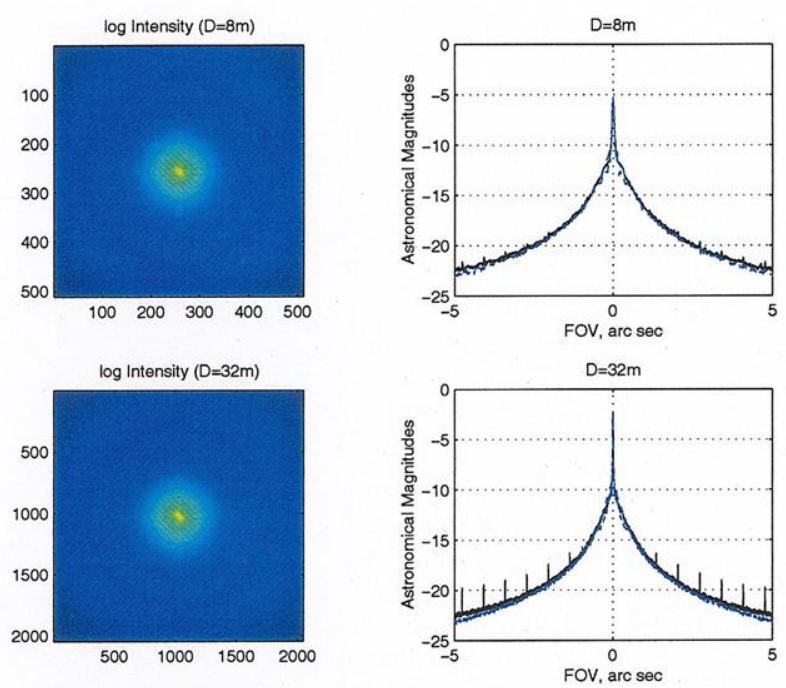

Figure 2. Sample MCAO-corrected point spread functions

This figure plots grayscales and one-dimensional profiles of H-band PSFs at the corner of a 1 arc minute square FOV of a sodium LGS MCAO system for telescope aperture diameters of 8 and 32 meters. The width of the images is 10.89 arc seconds. The solid (dashed) 1-dimensional profiles correspond to cuts at 0 and 90 degrees (45 and 135 degrees) through the images. See section 2 for information on the remaining MCAO system parameters.
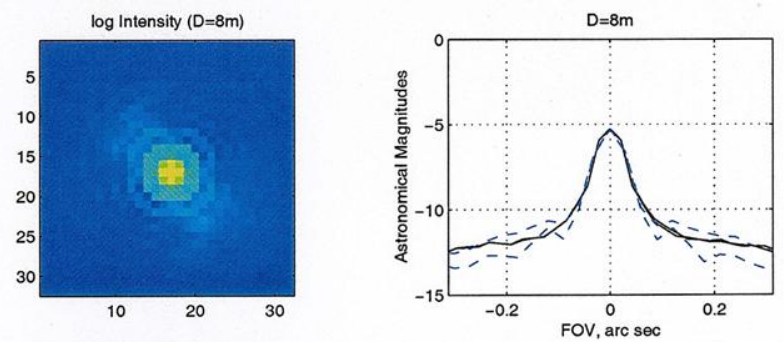

$\log$ Intensity $(D=32 m)$
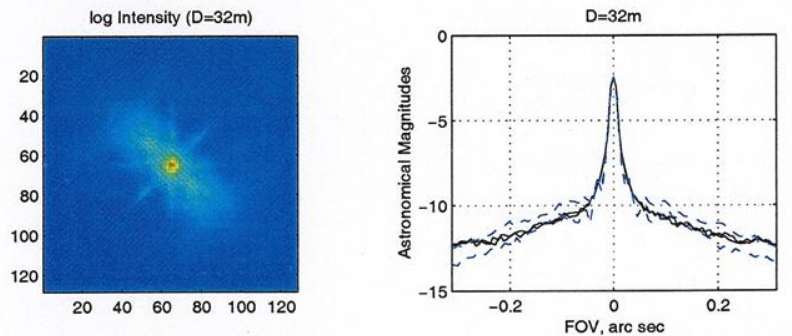

Figure 3. Central cores of sample MCAO-corrected point spread functions

This figure plots the central 0.68 arc second cores of the same MCAO-corrected PSFs appearing in Fig. 2 above.

using a single WFS measurement, not to iteratively correct residual errors with a significantly different statistical distribution.

Various approaches have already been developed for closed-loop wavefront reconstruction. These include (i) Kalman filtering, ${ }^{18,19}$ (ii) modal wavefront control, ${ }^{20}$ and (iii) variants of the MV reconstructor with constraints imposed that simplify its dynamic behavior in a closed-loop system. ${ }^{21}$ All of these existing methods 
require explict matrix multiplications, inversions, or eigenvector/eigenvalue decompositions, and therefore have computational requirements that scale as $D^{6}$. Alternative approaches with reduced computation requirements will probably need to be developed for very high-order MCAO systems on ELTs. ELTs: ${ }^{15}$

Thus far we have begun to investigate two new closed-loop wavefront reconstruction concepts for MCAO on

- Approximate closed-loop regularization (ACLR): The standard MV wavefront reconstruction algorithm is derived from (i) the second order statistics of atmospheric turbulence, (ii) the second-order statistics of WFS measurement noise, and (iii) the influence matrices relating atmospheric turbulence to phase distortion profiles and WFS measurements. Quantity (i) is sometimes referred to as the "regularization term:" Heuristically, it prevents the algorithm from reconstructing phase profiles that fit the WFS measurements precisely but are statistically very improbable. In principle, it should be possible to adapt the MV wavefront reconstruction algorithm to the closed-loop case by adjusting this regularization term to represent the second-order statistics of the residual phase errors left uncorrected by the AO control loop. However, computing the exact, steady-state value of this term requires the solution to a nonlinear matrix equation that is computationally intractable for a very high-order MCAO system. A very rough approximate solution may be determined by assuming that time delay is the dominant wavefront error source, but our first simulations using this approximation have not obtained stable results.

- Pseudo open-loop control (POLC): In principle, it should also be possible to apply the mimimum variance reconstruction algorithm in a closed-loop AO system by transforming closed-loop WFS measurements into the corresponding set of open-loop measurements that would have been recorded by an ideal WFS with a sufficiently large linear dynamic range. A heuristic description of this transformation is as follows:

1. Replace the WFS measurement vector $s$ by $s+G a$, where $a$ is the current DM actuator command vector and $G=\partial s / \partial s$ is the DM-to-WFS influence matrix.

2. Reconstruct a set of DM actuator commands $e$ to correct the total, open-loop phase distortion using the equation $e=R s$, where $R$ is the classical MV wavefront reconstruction algorithm.

3. Estimate the incremental DM actuator command needed to correct the residual closed-loop phase distortion as $\delta e=e-a$. (The value of $\delta e$ is equivalently, but less efficiently, given by the formula $\delta e=R s+(R G-I) a$.

The computational requirements for POLC are effectively the same as for the standard MV wavefront reconstruction algorithm, provided that the DM-to-WFS influence matrix $G$ is sparse. Note that this concept requires precise knowledge of the DM actuator command vector $a$ the DM-to-WFS influence matrix $G$ to accurately emulate the standard MV wavefront reconstruction algorithm.

Fig. 4 plots time histories of the performance of sample closed-loop sodium LGS MCAO system configurations using the POLC wavefront reconstruction algorithm. Results are presented for a time interval of 1.25 seconds (or 1000 simulation cycles) and aperture diameters of 8 and 32 meters. The RMS residual OPD averaged over the last 900 cycles has a value of about 151 and 162 nanometers, respectively, for the two aperture diameters. These residuals are quite close to the values of 149 and 159 nanometers reported in section 3 for the corresponding open-loop case. The simularity should not be surprising, given that (i) WFS measurement noise is negligible, (ii) previous simulations have indicated that a $800 \mathrm{~Hz}$ sampling rate and a $30 \mathrm{~Hz}$ control bandwidth is more than adequate for this set of atmospheric parameters and MCAO configuration (with $D=8 \mathrm{~m}$ ), ${ }^{6}$ and (iii) perfect knowledge of the DM actuator command vector and the DM-to-WFS influence matrix was available to the POLC reconstructor.

More work on this topic is clearly needed. We intend to continue experimenting with alternative regularization parameters to achieve stable results with the ACLR reconstruction algorithm, and to carefully evaluate the calibration and implementation issues associated with the POLC approach. 

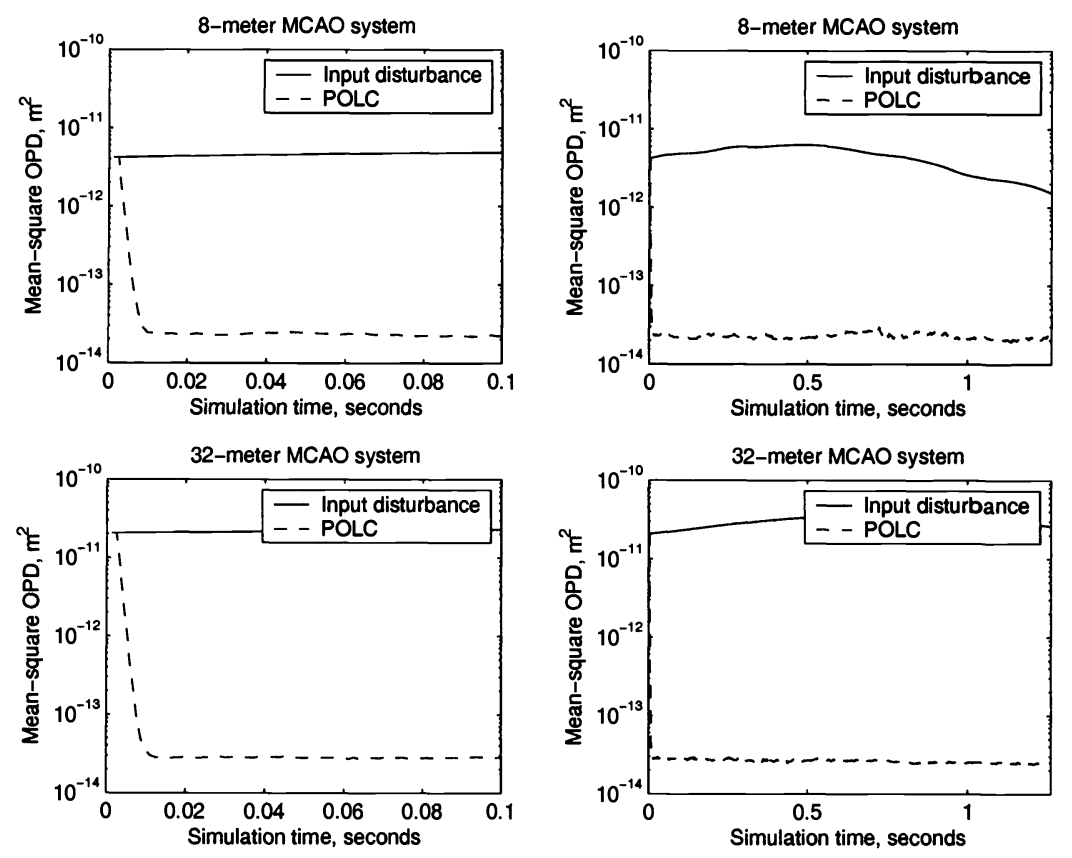

Figure 4. Closed-loop MCAO simulation results

This top [bottom] portion of this figure plots the time history of the mean-square residual OPD for an MCAO system using the POLC wavefront reconstruction algorithm on an 8-meter [32-meter] telescope. The results are plotted on two different timescales to illustrate both the convergence properties and long-term stability of the control loop. Please see section 2 for futher description of the MCAO system parameters.

\section{GUIDESTAR ELONGATION AND LGS SIGNAL REQUIREMENTS}

The simulation results presented in sections 3 and 4 above indicate that sodium laser guidestars are one potential approach to wavefront sensing for a MCAO system on a 30 meter class ELT. The mesospheric sodium layer is located at an altitude $H$ of about $90-95 \mathrm{~km}$, with a full width $h$ (to one significant digit) of about $10 \mathrm{~km}$. An individual subaperture in a Shack-Hartmann WFS observing a sodium LGS will actually image a streak of angular subtense $s h / H^{2}$, where $s$ is the separation between the subaperture and the laser launch telescope. Substituting $s=15 \mathrm{~m}$ for a 30-meter ELT yields an angle of about 2.3 arc seconds, compared with the 0.5-1.0 arc second spot size that is predicted to be achievable for an on-axis sodium LGS. These elongated images will significantly complicate WFS calibration, and also increase the LGS signal requirements for a specified level of WFS measurement noise.

At least three approaches for mitigating the impact of sodium LGS elongation have been suggested to date:

1. Higher resolution WFS focal planes and improved processing algorithms. Solar AO systems have already demonstrated excellent results by using a correlation track algorithm to estimate the differential image motion of a small patch of solar granulation as observed through a Shack-Hartmann subaperture. ${ }^{22}$ The same approach could be used with elongated sodium laser guidestars, since these images will contain some high spatial frequency content. LGS signal requirements would still increase compared with systems on smaller telescopes, but by a smaller amount than if a LGS WFS based upon quadrant detectors was used. At some point, using smaller and smaller pixels to resolve all of the structure in the sodium layer will become counter productive due to detector read noise. 
2. Multiple laser beams and laser launch telescopes per guidestar. Shack-Hartmann WFS measurement accuracy will not, in principle, be degraded for image motion in the direction perpendicular to spot elongation. The direction of elongation is always parallel to the separation between the subaperture and the laser launch telescope (LLT). By using three LLTs in different locations, it is possible to generate a triplet of guidestars that provide at least two independent directions of image elongation on all subapertures. This enables accurate measurements of $x$ - and $y$-image motion on all subapertures with an integrated LGS signal level no larger than a factor of two greater than would be required if guidestar elongation was not an issue, albeit at the expense of additional complications to the LGS facility and wavefront sensors. ${ }^{16}$

3. In principle, it should be possible to track a short ( $1 \mathrm{~km}$ or less) laser pulse through the sodium layer by dynamically refocusing the LGS wavefront sensor. This approach will eliminate LGS elongation entirely if a guide star laser with a suitable pulse format can be developed.

At this point we have begun evaluating the LGS signal requirements associated with approach number 1, since approach 2 has already been studied and approach 3 is largely a matter of developing new hardware.

An upper bound on the performance of any image motion estimation algorithm is defined by the matched filtering method. Assume that the image displacements $\theta$ are small enough that the vector of pixel counts $I$ formed by the image on the focal plane is modeled by the first-order approximation

$$
I=I_{0}+G \theta+n,
$$

where $I_{0}$ is the vector of pixel counts with no image motion or measurement noise, $G=\partial I / \partial \theta$ is the Jacobian matrix describing the first-order impact of image motion on the pixel counts, and $n$ is the variability in the pixel counts due to detector read noise and photon statistics. The matched filter estimation algorithm is defined by the formula

$$
\widehat{\theta}=\left(G^{T} \Sigma_{n}^{-1} G\right)^{-1} G^{T} \Sigma_{n}^{-1}\left(I-I_{0}\right),
$$

where $\Sigma_{n}=\left\langle n n^{T}\right\rangle$ is the covariance matrix describing the second-order statistics of the noise in the pixel counts. Assuming that the image $I$ is expressed in units of photodetection events,

$$
\Sigma_{n}=\operatorname{diag}\left(I_{0}+\sigma_{e}^{2}\right),
$$

where $\sigma_{e}$ is the RMS detector read noise. Provided that the linear model for $I$ given at the beginning of this paragraph is valid, the covariance matrix $\Sigma_{\theta}=\left\langle(\theta-\widehat{\theta})(\theta-\widehat{\theta})^{T}\right\rangle$ describing the second-order statistics of the image motion estimation errors for the matched filter algorithm takes the value

$$
\Sigma_{\theta}=\left(G^{T} \Sigma_{n}^{-1} G\right)^{-1}
$$

The RMS $x$ - and $y$-components of the image estimation error are the square roots of the diagonal of $\Sigma_{\theta}$.

We have computed this performance bound for a Shack-Hartmann WFS subaperture observing an extended sodium laser guidestar. The nominal image $I_{0}$ is modeled as the convolution of three terms: The two-dimension laser irradiance pattern in the sodium layer, the subaperture image of an ideal point source, and the onedimensional profile of the sodium layer density. The first two terms have been computed using Fried's formula for the short-exposure (tilt-removed) MTF of atmospheric turbulence, and the three sample sodium layer profiles illustrated in Fig. 5 have been taken from the Gemini sodium layer measurement campaign at Cerro Pachon. ${ }^{23}$

Fig. 6 plots the matched filter bound on the image motion estimation error in the direction of spot elongation for the "intermediate" sodium layer profile and a variety of WFS parameters:

- A 4 meter separation between the LLT and the WFS subaperture, 5 electrons of read noise, and a quad cell composed of $2 \times 21$ arc second detectors. This is the nominal case for the Gemini-South LGS AO system design, and the impact of spot elongation is relatively modest.

- A 15 meter LLT-to-subaperture separation, 5 electrons of read noise, and a 6-arc-second-square subaperture FOV imaged on either $0.5,1$, or 3 arc second pixels. 


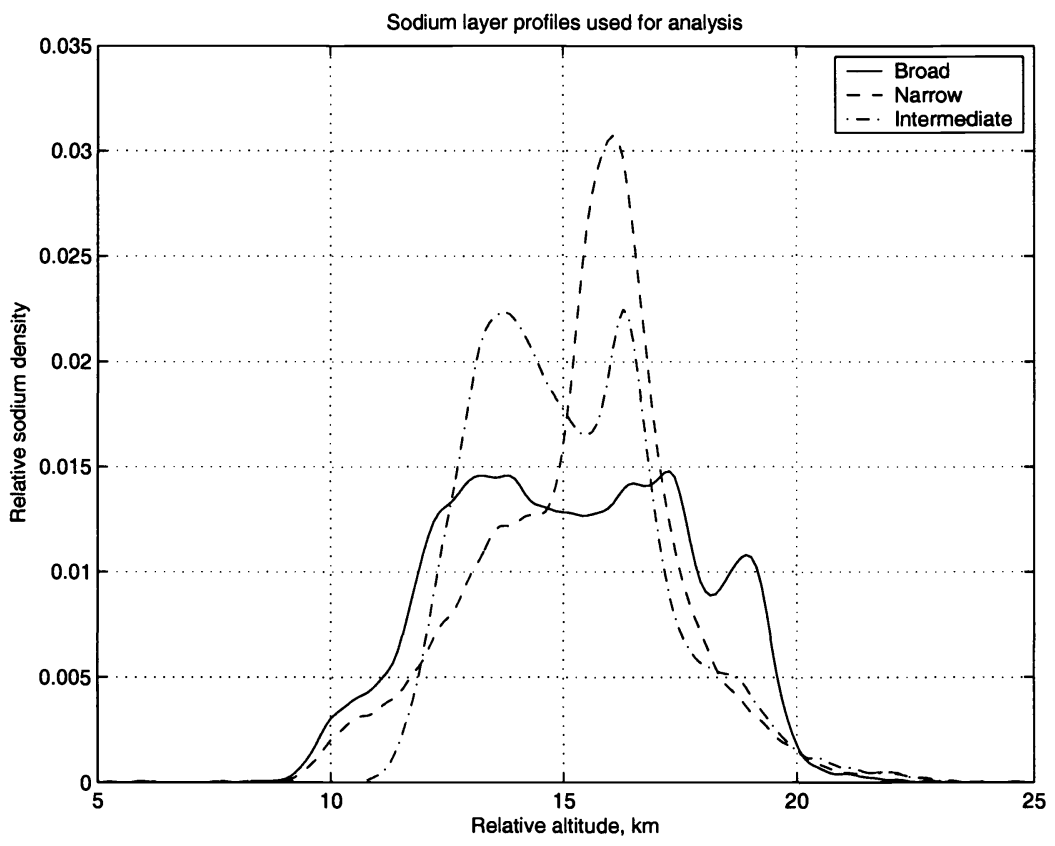

Figure 5. Sample sodium layer density profiles

This figure plots three sodium layer density profiles obtained from the Gemini measurement campaign at Cerro Pachon. No statistical analysis of the profile characteristics has been performed, but some effort has been made to select representative broad, narrow, and intermediate profiles.

It is clear from Fig. 6 that sodium LGS elongation will significantly increase the LGS signal requirements for a 30-meter class telescope no matter what parameters are assumed for the WFS focal plane. However, reducing the pixel size from 3 to 1 arc seconds reduces the LGS signal requirement by about 15 per cent if the desired WFS measurement noise is $0.03 \mathrm{arc} \mathrm{sec}$ or less. Further reducing the pixel size to 0.5 arc seconds is counterproductive, since the improved resolution of the spot is offset by the increased impact of detector read noise. This penalty can be reduced by assuming that detectors with only 3 read noise electrons will be feasible, but it appears that the noise-optimal pixel size is still approximately 1 arc second.

Using the results in Fig. 6 (and analogous results for the remaining two sodium profiles), we can now determine how LGS signal requirements compare on 30- and 8-meter class telescopes for the same level of RMS LGS WFS measurement noise at the edge of the aperture. Assuming 5 electrons of read noise, LGS signal requirements are greater by slightly less than a factor of 3 for the larger telescope. This can be reduced to a factor of about 2.25 if we are willing to assume that detectors with 3 electrons of read noise will be available.

These initial results should only viewed as establishing an upper bound on laser power requirements, since the magnitude of the guidestar elongation varies significantly over the 30-meter aperture and we have picked the worst point to make the comparison. For a more accurate performance estimate, the closed-loop MCAO simulations described in section 4 should be repeated using spatially varying WFS measurement noise statistics derived for a particular laser power, sodium layer profile, and set of WFS detector parameters. We expect to be able to report on the results of such simulations in the near future.

\section{ACKNOWLEDGMENTS}

The New Initiatives Office is a partnership between two divisions of the Association of Universities for Research in Astronomy (AURA), Inc.: The National Optical Astronomy Observatory (NOAO) and the Gemini Obser- 


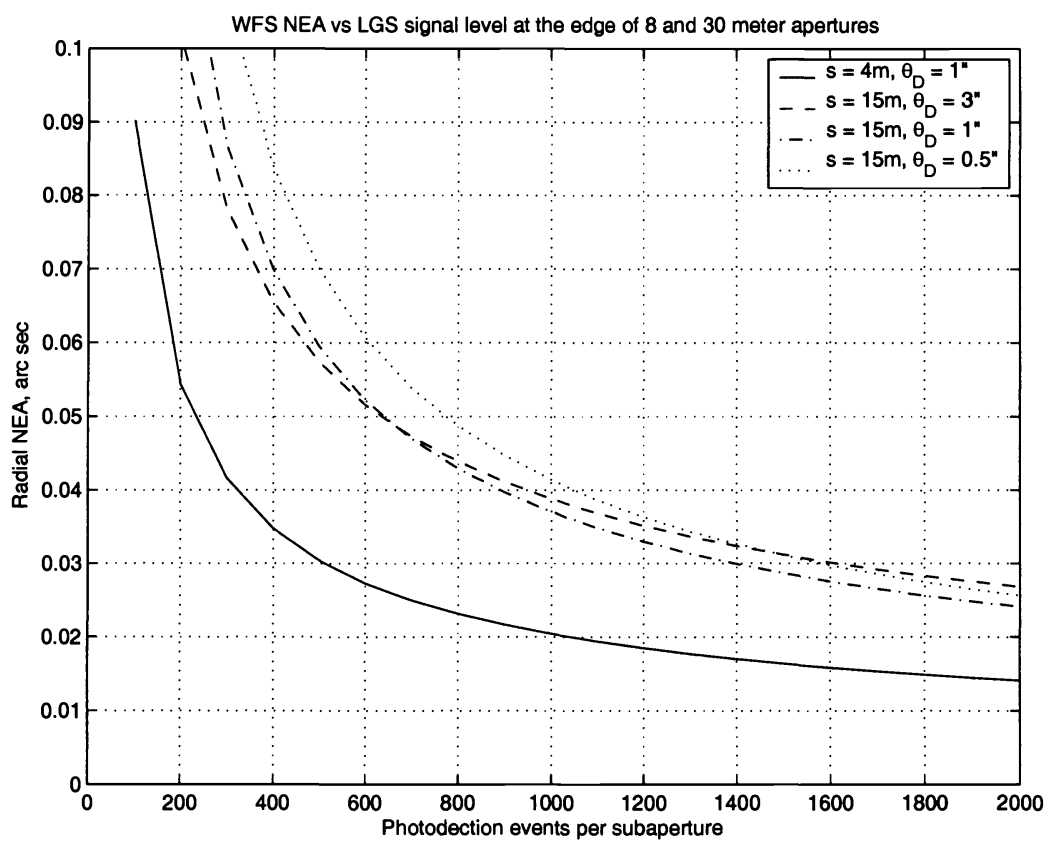

Figure 6. LGS WFS image motion estimation error vs. LGS signal level

This figure plots the LGS WFS NEA in the direction of guidestar elongation as a function of the LLT-to-subaperture separation $s$ and angular subtense $\theta_{D}$ of the WFS focal plane pixels. The calculations assume a matched filter image motion estimation algorithm, the "intermediate" sodium layer profile in Fig. 5, and 5 electrons of detector read noise.

vatory. NOAO is operated by AURA under a cooperative agreement with the National Science Foundation (NSF). The Gemini Observatory is operated by AURA under a cooperative agreement with the NSF on behalf of the Gemini partnership: the National Science Foundation (United States), the Particle Physics and Astronomy Research Council (United Kingdom), the National Research Council (Canada), CONICYT (Chile), the Australian Research Council (Australia), CNPq (Brazil) and CONICET (Argentina).

\section{REFERENCES}

1. J. M. Beckers, "Increasing the size of the isoplanatic patch with multi-conjugate adaptive optics," in Proceedings of European Southern Observatory Conference and Workshop on Very Large Telescopes and Their Instrumentation, M.-H. Ulrich, ed., Vol. 30 of ESO Conference and Workshop Proceedings (European Southern Observatory, Garching, Germany, 1988), pp. 693-703.

2. J. E. Nelson, "CELT Rocks," in Future Giant Telescopes, James Roger P. Angel and Roberto Gilmozzi, ed., SPIE Proceedings Vol. 4840 47-59 (2002).

3. S. E. Strom, L. M. Stepp, M. Mountain, and B. Gregory, "Giant segmented mirror telescope: a point design based on science drivers," in Future Giant Telescopes, James Roger P. Angel and Roberto Gilmozzi, ed., SPIE Proceedings Vol. 4840 116-128 (2002).

4. P. Dierickx, J. Beckers, E. Brunetto, R. Gilmozzi, F. Koch, E. Marchetti, G. J. Monnet, M. Quattri, M. S. Sarazin, J. Spyromilio, and N. Yaitskova, "Eye of the beholder: designing the OWL," in Future Giant Telescopes, James Roger P. Angel and Roberto Gilmozzi, ed., SPIE Proceedings Vol. 4840 151-170 (2002).

5. J. R. P. Angel, J. H. Burge, J. L. Codona, W. B. Davison, and B. Martin, "20- and 30-m telescope designs with potential for subsequent incorporation into a track-mounted pair (20/20 or 30/30)," in Future Giant Telescopes, James Roger P. Angel and Roberto Gilmozzi, ed., SPIE Proceedings Vol. 4840 183-193 (2002). 
6. Gemini-South MCAO PDR documentation, http://www.gemini.edu/sciops/instruments/adaptiveOptics/AOarchive.html

7. E. Marchetti, N. N. Hubin, E. Fedrigo, J.Brynnel, B. Delabre, R. Donaldson, F. Franza, R. Conan, M. Le Louarn, C. Cavadore, A. Balestra, D. Baade, J.-L.Lizon, R. Gilmozzi, G. J. Monnet, R. Ragazzoni, C. Arcidiacono, A. Baruffolo, E. Diolaiti, J. Farinato, E. Vernet-Viard, D.J. Butler, S. Hippler, and A. Amorin, "MAD: the ESO multiconjugate adaptive optics demonstrator," in Adaptive Optical Systems Technologies II, Peter L. Wizinowich, ed., SPIE Proceedings Vol. 4839 317-328 (2002).

8. M. Lloyd-Hart and N. M. Milton, "Design and expected performance of the 6.5-m MMT MCAO system," in Adaptive Optical Systems Technologies II, Peter L. Wizinowich, ed., SPIE Proceedings Vol. 4839 578-587 (2002).

9. B. L. Ellerbroek, "Wave optics propagation code for multiconjugate adaptive optics," in Adaptive Optics System Technology II, SPIE Proceedings Vol. 4494 104-121 (2001).

10. A. Ahmadia and B. L. Ellerbroek, "Parallelized simulation code for multiconjugate adaptive optics," in Astronomical Adaptive Optics Systems and Applications, R. K. Tyson and M. Lloyd-Hart, ed., SPIE Proceedings Vol. 5169 (in press).

11. B. L. Ellerbroek, L. Gilles, and C. R. Vogel, "Computationally efficient wavefront reconstruction algorithms for simulations of multiconjugate adaptive optics on giant telescopes," in Adaptive Optical Systems Technologies II, Peter L. Wizinowich, ed., SPIE Proceedings Vol. 4839 989-1000 (2002).

12. L. Gilles, B. L. Ellerbroek, and C. R. Vogel, "Layer-oriented multigrid wavefront reconstruction algorithms for multiconjugate adaptive optics," in Adaptive Optical Systems Technologies II, Peter L. Wizinowich, ed., SPIE Proceedings Vol. 4839 1011-1022 (2002).

13. L. Gilles, B. L. Ellerbroek, and C. R. Vogel, "Order N sparse minimum-variance open-loop reconstructor for extreme adaptive optics," Optics Letters 28 (2003).

14. B. L. Ellerbroek, L. Gilles, and C. R. Vogel, "Numerical Simulations of Multi-Conjugate Adaptive Optics Wavefront Reconstruction on Giant Telescopes," Appl. Opt. 42 4811-4818 (2003).

15. B. L. Ellerbroek, L. Gilles, and C. R. Vogel, "Simulations of closed-loop wavefront reconstruction algorithms for multiconjugate adaptive optics on giant telescopes," in Astronomical Adaptive Optics Systems and Applications, R. K. Tyson and M. Lloyd-Hart, ed., SPIE Proceedings Vol. 5169 (in press).

16. J. Nelson, "Laser Beacon Spot Elongation," CfAO 2003 Spring Retreat, http://cfao.ucolick.org/presentations/springretreat2003/SR03 Nelson LaserBeacon.pdf.

17. J. Vernin, A. Agabi, R. Avila, M. Azouit, R. Conan, F. Martin, E. Masciadri, L. Sanchez, and A. Ziad, "1998 Gemini site testing campaign: Cerro Pachon and Cerro Tololo," Gemini Doc. RTP-AO-G0094 (Gemini Observatory, Hilo, Hawaii, 2000).

18. B. Le Roux, J. M. Conan,C. Kulcsar, H-F. Raynaud, L. M. Mugnier, T. Fusco, "Optimal control law for multi-conjugate adaptive optics," in Adaptive Optical Systems Technologies II, Peter L. Wizinowich, ed., SPIE Proceedings Vol. 4839 878-889 (2002).

19. D. T. Gavel and W. Wiberg, "Towards strehl-optimizing adaptive optics controllers," in Adaptive Optical Systems Technologies II, Peter L. Wizinowich, ed., SPIE Proceedings Vol. 4839 890-901 (2002).

20. E. Gendron and P. Lena, "Astronomical adaptive optics i. modal control optimization," Astronomy and Astrophysics 291, 337-347 (1994).

21. B. L. Ellerbroek, "First order performance evaluation of adaptive-optics systems for atmospheric turbulence compensation in extended field-of-view astronomical telescopes," J. Opt. Soc. Am. A 11, 783-805 (1994).

22. T. R. Rimmele, K. Richards, S. L .Hegwer, D. Ren, S. Fletcher, S. Gregory, L. V. Didkovsky, C. J. Denker, W. Marquette, J. Marino, and P. R. Goode, "Solar adaptive optics: a progress report," in Adaptive Optical Systems Technologies II, Peter L. Wizinowich, ed., SPIE Proceedings Vol. 4839 635-646 (2002).

23. C. d'Orgeville, F. R. Rigaut, M. Boccass, C. Dainty, E. Figueroa, R. Flicker, B. Gregory, L. Michaille, J. C. Quartel, A. A. Tokovinin, G. Trancho, and N. J. Wooder, "Preliminary results of the 2001-2002 Gemini sodium monitoring campaign at Cerro Tololo, Chile," in Adaptive Optical Systems Technologies II, Peter L. Wizinowich, ed., SPIE Proceedings Vol. 4839 492-503 (2002). 